\title{
Response of Seeds, Oil Yield and Fatty Acids Percentage of Jojoba Shrub Strain EAI to Mycorrhizal Fungi and Moringa Leaves Extract
}

\author{
Amira K. G. Atteya ${ }^{1, *(D)}$, Rokayya Sami ${ }^{2, *(D)}$, Amina A. M. Al-Mushhin ${ }^{3}$, Khadiga Ahmed Ismail ${ }^{4}$ (D) \\ and Esmail A. E. Genaidy ${ }^{5}$ (iD)
}

1 Horticulture Department, Faculty of Agriculture, Damanhour University, Damanhour 22516, Egypt

2 Department of Food Science and Nutrition, College of Sciences, Taif University, Taif 21944, Saudi Arabia

3 Department of Biology, College of Science and Humanities in Al-Kharj, Prince Sattam Bin Abdulaziz University, Al-Kharj 11942, Saudi Arabia; a.almushhin@psau.edu.sa

4 Department of Clinical Laboratory Sciences, College of Applied Medical Sciences, Taif University, Taif 21944, Saudi Arabia; khadigaah.aa@tu.edu.sa

5 Pomology Department, National Research Centre, Giza 12622, Egypt; esmail_nrc@yahoo.com

* Correspondence: amira.khames@agr.dmu.edu.eg (A.K.G.A.); rokayya.d@tu.edu.sa (R.S.)

check for updates

Citation: Atteya, A.K.G.; Sami, R.; Al-Mushhin, A.A.M.; Ismail, K.A.; Genaidy, E.A.E. Response of Seeds, Oil Yield and Fatty Acids Percentage of Jojoba Shrub Strain EAI to Mycorrhizal Fungi and Moringa Leaves Extract. Horticulturae 2021, 7, 395. https://doi.org/10.3390/ horticulturae7100395

Academic Editors: Othmane Merah, Purushothaman Chirakkuzhyil Abhilash, Magdi T. Abdelhamid, Hailin Zhang and Bachar ZEBIB

Received: 17 September 2021

Accepted: 10 October 2021

Published: 13 October 2021

Publisher's Note: MDPI stays neutral with regard to jurisdictional claims in published maps and institutional affiliations.

Copyright: (c) 2021 by the authors. Licensee MDPI, Basel, Switzerland. This article is an open access article distributed under the terms and conditions of the Creative Commons Attribution (CC BY) license (https:/ / creativecommons.org/licenses/by/ $4.0 /)$.
Abstract: Jojoba seeds have a unique storage lipid wax which is suitable as a basic feedstock in the chemical industry. For saving both human health and the environment, there is a continuous need to search for alternative safe natural sources of plant nutrients. Therefore, in this study the effect of mycorrhizal fungi and Moringa oleifera leaves extract on growth, flowering, fruits set, yield and the chemical composition of the jojoba shrub was studied. The application of a combination of treatments of $20 \mathrm{~g} \mathrm{~L}^{-1}$ mycorrhizal fungi plus $30 \mathrm{~g} \mathrm{~L}^{-1}$ Moringa oleifera leaves extract recorded the maximum mean values of main branch length, length of secondary branches, number of branched nodes, number of secondary branches, flowering percentage, final fruit set percentage, seeds yield per shrub and per hectare, percentage of minerals, proteins as well as oil yield per shrub and per hectare, chlorophyll a and b, N, P, K percentage with a minimum mean value of the number of days until full bloom in both seasons. The maximum percentage of Gadoleic fatty acid was found with the combination treatment of uninoculation plus $10 \mathrm{~g} \mathrm{~L}^{-1}$ Moringa oleifera leaves extract.

Keywords: growth; flowering; fruits set; jojoba seeds yield; jojoba oil yield; fatty acids; Gadoleic acid; mycorrhizal fungi; Moringa oleifera leaves extract

\section{Introduction}

Jojoba (Simmondsia chinensis) is a member of the family Simmondsiaceae. It is endemic to the Sonoran Desert in Southern Arizona, Southern California and Northern Mexico [1]. Cultivation of the jojoba shrub has been established in many desert and semi-desert areas. Its mature seeds are a hard oval, dark brown in color and represent the economic part of the jojoba shrub [2]. Jojoba seeds have a unique storage lipid wax consisting of esters formed from acids and alcohols [3]. Jojoba oil is distinguished by its lack of odor, purity and heat-resistant lubricating properties. Therefore, it is suitable as a basic feedstock in the chemical industry [1] such as pharmaceuticals, lubricants, gear additives, extenders, anti-foaming agents as well as in the wax and polish industries [3,4].

There are a lot of factors that control the growth, flowering and fruiting of jojoba shrubs and their seed yield and consistency, such as plant growth regulators and nutrients $[5,6]$. Fertilizers are any organic or inorganic material of natural or synthetic origin that is added to soil to supply one or more plant nutrients essential for plant growth. On the other hand, the use of inorganic fertilizers is not only high cost, but is also associated with environmental pollution and soil degradation. In recent decades, the innovative view of agricultural production is attracting the increasing demand for bio-organic fertilizers $[7,8]$. 
Thus, agricultural farming practices have been evolving towards environmental friendly systems. It means the reduction in chemical inputs without decreasing the quantity or quality of crop yield.

Mycorrhizal fungi can be integrated with soil management to achieve low-cost sustainable agricultural systems [9]. They are used as biofertilizers which constitute a group of root obligate biotrophs that exchange mutual benefits with about $80 \%$ of plants [10-13]. The host plants perform the photosynthesis process and supply the fungus with soluble carbon. On the other hand, the fungus increases the surface contact of the roots with the soil and promotes plant growth, as it enhances the uptake of immobile nutrients such as phosphorus, nitrogen, copper and zinc as well as increasing water absorption [14,15]. The elements are absorbed through the fungus by a specific absorption system and transferred to the plant via the innate contact appendages and intracellular structures; it is assumed that inducing the transfer of nutrients during the symbiosis process enhances the movement of nutrients to the plant. In addition, mycorrhizal fungi increase the resistance to drought and salinity stresses as well as pests and soil-borne diseases and improve growth and osmotic adjustment through stimulating the production of growth-regulating substances [16]. In this field, many studies such as those by Davies et al. [17] on pepper, Ruiz-Lozano and Azcon [18] on lettuce, Auge et al. [19] on the common bean, Ruiz-Lozano et al. [20] on soybean, Ortas [21] on maize, wheat and cotton, Karandashov and Bucher [22], Roldan et al. [23] on Juniperus, Ortas and Rattan [24] on pepper, wheat and maize, Tobar et al. [25] on lettuce, Igiehon et al. [26] on soybean, Jabborova et al. [27] on spinach, Jabborova et al. [28] on ginger and Zewail et al. [29] on stevia were conducted.

Moringa (Moringa oleifera $\mathrm{L}$.) belongs to the Moringaceae family. Moringa leaves extract is a rich source of macro and micronutrients, amino acids, vitamins, ascorbates, phenolic compounds antioxidants and growth-regulating hormones such as auxins, cytokinins, gibberellins, jasmonic and salicylic acids [30-34]. Thus, it possesses the potential to promote plant growth; hence, it can be used as a natural plant growth promotor. The studies by El-Serafya and El-Sheshtawy [34] on fennel, Prabhu et al. [35] on basil, Ali et al. [36] on geranium and Ahmad et al. [37] on freesia reported that using Moringa oleifera leaves extract as a biostimulant could enhance plant growth, yield and chemical composition of plants.

Using organic agriculture regulations for medicinal and aromatic plant cultivation increases Egyptian trade with European Union [38]. To the best of our knowledge, no reports have been conducted on the combined impact of mycorrhizal fungi and Moringa oleifera leaves extract on jojoba shrubs. Therefore, the aim of this study was to study the influence of mycorrhizal fungi and Moringa oleifera leaves extract as well as their combination treatments on the growth, yield and chemical composition of the jojoba shrub (Strain EAI) under an organic agriculture system.

\section{Materials and Methods}

This experiment was conducted to find out the effect of ground inoculation of arbuscular mycorrhizal fungi inoculums of Glomus mosseae and Glomus fasciculatum and foliar application of Moringa oleifera leaves extract and on jojoba shrubs (Simmondsia chinensis), at a private farm in the EL-Behira governorate, Egypt. Arbuscular mycorrhizal fungi were obtained from the Agriculture Research Center in Egypt. A total of $20 \mathrm{~g} \mathrm{~L}^{-1}$ arbuscular mycorrhizal fungi (approximately 2400 spores) were added as a ground addition. Each shrub was given $3 \mathrm{~L}$ at the beginning of every season. Jojoba shrubs of similar vigor, age (twelve years old) and size were selected for the application of studied treatments during the two studied seasons (2018/2019 and 2019/2020). The experiment laid out is a factorial arranged in randomized complete block design (RCBD) with three replications. For each replication of treatment, the same shoot concerning height, thickness, vigor and number of fruit and orientation was selected. Distances between rows and within shrubs in rows were 3 and $2.5 \mathrm{~m}$, respectively. A drip irrigation system was applied in the orchard, weed, pest control and fertilization conducted following the standard agro-management practices. 
The orchard soil analysis is given in (Table 1), and water irrigation analyses are given in (Table 2) according to the procedures.

Table 1. Some physical and chemical analyses of the orchard soil.

\begin{tabular}{cccccccccc}
\hline Parameters & Texture Class & $\mathbf{E C}\left(\mathbf{d S m}^{-1}\right)$ & $\mathbf{C a}^{++}$ & $\mathbf{M g}^{++}$ & $\mathbf{N a}^{+}$ & $\mathbf{K}^{+}$ & $\mathbf{H C O}_{3}^{-}$ & $\mathbf{C l}^{-}$ & $\mathbf{S O}_{4}{ }^{2-}$ \\
\hline Values & sandy & 2.28 & 2.15 & 3.75 & 19.57 & 0.35 & 1.13 & 21.21 & 4.57 \\
\hline
\end{tabular}

Table 2. Chemical characteristics of irrigation water used for the present study.

\begin{tabular}{cccccccccc}
\hline Parameters & $\mathbf{p H}$ & $\mathbf{E C}\left(\mathbf{d S m}^{-1}\right)$ & $\mathbf{C a}^{++}$ & $\mathbf{M g}^{++}$ & $\mathbf{N a}^{+}$ & $\mathbf{K}^{+}$ & $\mathbf{H C O}_{3}{ }^{-}$ & $\mathbf{C l}^{-}$ & $\mathbf{S O}_{4}{ }^{2-}$ \\
\hline Values & 7.44 & 3.43 & 5.34 & 3.66 & 21.72 & 3.25 & 1.96 & 29.22 & 3.23 \\
\hline
\end{tabular}

\subsection{Moringa Oleifera Leaves Extract Preparation}

For preparing aqueous extract solutions of Moringa oleifera leaves, the plant material was dried in an oven at $70^{\circ} \mathrm{C}$ for $48 \mathrm{~h}$. Then, the dried material was ground in a grinder and passed through a 40-mesh screen. To prepare the extracts, $100 \mathrm{~g}$ of each ground plant material were macerated in $1000 \mathrm{~mL}$ distilled water. Solutions were placed in an orbital shaker at room temperature for $24 \mathrm{~h}$. The extracts were filtered using Whatman filter paper No.1 [39]. The obtained extracts were diluted in order to achieve the concentrations. The analysis of Moringa leaves extract is shown in Table 3. This extract was used to achieve the different analyses and treatments. Extract analyses are given in (Table 3).

Table 3. Chemical characteristics of Moringa oleifera leaves extract used for the present study.

\begin{tabular}{ccc}
\hline Parameters & Units & Values \\
\hline Vitamin C & $\mathrm{mg} \mathrm{g}^{-1} \mathrm{FW}$ & 2.63 \\
Total phenolic content & $\mathrm{mg} \mathrm{Gallic}^{-1} \mathrm{FW}$ & 2.24 \\
Total flavonoid content & $\mathrm{mg} \mathrm{Rutin}^{-1} \mathrm{FW}$ & 1.22 \\
Antioxidant activity determinations $\mathrm{IC}_{50}$ & $\mu \mathrm{g} \mathrm{mL}^{-1}$ & 176 \\
$\mathrm{~N}$ & $\%$ & 3.52 \\
$\mathrm{P}_{2} \mathrm{O}_{5}$ & $\%$ & 0.252 \\
$\mathrm{~K}_{2} \mathrm{O}$ & $\%$ & 2.87 \\
\hline
\end{tabular}

\subsection{Treatments}

Arbuscular mycorrhizal fungi at 0 and $20 \mathrm{~g} \mathrm{~L}^{-1}$ was added to soil in the beginning of the experiment. For fungi inoculate, $3 \mathrm{~L}$ containing $20 \mathrm{~g} \mathrm{~L}^{-1}$ arbuscular mycorrhizal fungi (approximately 2400 spores $\mathrm{L}^{-1}$ ) were added as ground addition for every shrub at the beginning of every season (at the beginning of December).

Treatments of Moringa leaves extract were performed by spraying $4 \mathrm{~L}$ per tree every two weeks from the beginning of December. Moringa oleifera leaves treatments were 0, 10, 20, $30 \mathrm{~g} \mathrm{~L}^{-1}$ leaves extract. The combined treatments are presented in Table 4.

Table 4. The combination treatments of arbuscular mycorrhizal fungi and aqueous extract solutions of Moringa oleifera leaves extract.

\begin{tabular}{ccc}
\hline Treatments & Arbuscular Mycorrhizal Fungi & Moringa oleifera Leaves Extract \\
\hline Control & Without arbuscular mycorrhizal & $0 \mathrm{~g} \mathrm{~L}^{-1}$ \\
T1 & fungi $\left(0 \mathrm{~g} \mathrm{~L}^{-1}\right)$ & $10 \mathrm{~g} \mathrm{~L}^{-1}$ \\
T2 & & $20 \mathrm{~g} \mathrm{~L}^{-1}$ \\
T3 & & $30 \mathrm{~g} \mathrm{~L}^{-1}$ \\
\hline T4 & With arbuscular mycorrhizal fungi & $0 \mathrm{~g} \mathrm{~L}^{-1}$ \\
T5 & $\left(20 \mathrm{~g} \mathrm{~L}^{-1}\right)$ & $10 \mathrm{~g} \mathrm{~L}^{-1}$ \\
T6 & & $20 \mathrm{~g} \mathrm{~L}^{-1}$ \\
T7 & & $30 \mathrm{~g} \mathrm{~L}^{-1}$ \\
\hline
\end{tabular}




\subsection{Parameters}

For each treatment, nine shrubs (three shrubs for every replicate) were tagged by random selection.

\subsubsection{Vegetative and Reproductive Measurements Branch Characteristics}

Branch length $(\mathrm{cm})$, length of secondary branches per every branch $(\mathrm{cm})$, number of nodes forming branches per meter, number of secondary branches on the main branch per meter.

\section{Flowering, Fruiting and Seed Yield}

Three branches per tree from each treatment were tagged in December 2018 and 2019, and the full bloom date, flowering percentage, final fruit set percentage and seed yield ( $\mathrm{g}_{\text {tree }}{ }^{-1}$ and $\mathrm{kg} \mathrm{ha}^{-1}$ ) were recorded as mentioned by Atteya et al. [6].

\subsubsection{Chemical Analyses of Seed}

Jojoba oil was extracted from seeds with the Soxhlet extraction method with hexane solvent, and it was calculated as oil percentage (\%) and yield per hectare $\left(1 \mathrm{ha}^{-1}\right)$. Additionally, crude protein (\%), mineral (\%) and total carbohydrate (\%) were performed following the AOAC [40]. The seeds' fixed oil analyses were performed as mentioned by Atteya et al. [6].

\subsubsection{Chlorophyll $a$ and $b$}

They were determined according to Wintermans and Mats [41].

\subsubsection{The Macro Elements (N, P and K)}

They were determined in leaves according to the method of Chapman and Pratt [42].

\subsection{Statistical Analysis}

Analysis of variance with SAS software [43] was carried out on the test treatment data. Treatments' means were compared using the LSD test at $5 \%$ level of probability. Data in Tables are mean value \pm SE.

\section{Results}

\subsection{Growth Parameters}

Vegetative parameters of jojoba shrubs varied according to ground inoculation of arbuscular mycorrhizal fungi and foliar application of Moringa oleifera leaves extract and their interaction. Tables 5 and 6 indicates that jojoba shrubs treated with arbuscular mycorrhizal fungi recorded an increment in the main branch length (19 and 20\%), secondary branch length ( 40.6 and $40.3 \%$ ), number of branched nodes (27.42 and $27.69 \%$ ) and number of secondary branches ( 35.62 and $35.48 \%$ ) compared with corresponding untreated control shrubs in both seasons. With comparing the spraying levels of Moringa oleifera leaves extract, the application of $30 \mathrm{~g} \mathrm{~L}^{-1}$ gave the maximum significant mean values of shrub growth parameters with increment percent of 35 and 35\% for the main branch length, 64 and $64 \%$ for secondary branch length, 42 and $41 \%$ for the number of branched nodes and 53 and 53\% for the number of secondary branches compared with control shrubs. The interaction was significant between Moringa oleifera leaves extract and ground inoculation of arbuscular mycorrhizal fungi treatments. Moreover, the maximum significant mean values of jojoba shrub growth were found with the combination treatment of arbuscular mycorrhizal fungi $\left(20 \mathrm{~g} \mathrm{~L}^{-1}\right)$ plus $30 \mathrm{~g} \mathrm{~L}^{-1}$ Moringa oleifera leaves extract in both seasons, as this treatment recorded an increment percentage (57 and 58\%), (110 and 111\%), (73 and $72 \%),(92$ and $93 \%$ ) for main branch length, secondary branch length, number of branched nodes and number of secondary branches, respectively, compared with control treatment in both studied seasons. 
Table 5. The mean values of the main branch length $(\mathrm{cm})$, length of secondary branches $(\mathrm{cm})$ and number of branched nodes of jojoba shrubs affected with ground inoculation of arbuscular mycorrhizal fungi and foliar application of Moringa oleifera leaves extract, as well as their combination treatments in both seasons of the study.

\begin{tabular}{|c|c|c|c|c|c|c|}
\hline \multirow{2}{*}{ Treatment } & \multicolumn{2}{|c|}{ Main Branch Length (cm) } & \multicolumn{2}{|c|}{ Length of Secondary Branches (cm) } & \multicolumn{2}{|c|}{ Number of Branched Nodes } \\
\hline & 1st Season & 2nd Season & 1st Season & 2nd Season & 1st Season & 2nd Season \\
\hline \multicolumn{7}{|c|}{ Arbuscular mycorrhizal fungi $\left(\mathrm{g} \mathrm{L}^{-1}\right)$} \\
\hline Control & $84 \pm 10 b$ & $86 \pm 10 b$ & $28.6 \pm 5.2 b$ & $29.5 \pm 5.3 b$ & $2.99 \pm 0.30 b$ & $3.07 \pm 0.30 \mathrm{~b}$ \\
\hline 20 & $100 \pm 11 \mathrm{a}$ & $103 \pm 12 \mathrm{a}$ & $40.2 \pm 7.8 \mathrm{a}$ & $41.4 \pm 8.0 \mathrm{a}$ & $3.81 \pm 0.63 \mathrm{a}$ & $3.92 \pm 0.65 \mathrm{a}$ \\
\hline \multicolumn{7}{|c|}{ Moringa oleifera leaves extract $\left(\mathrm{g} \mathrm{L}^{-1}\right)$} \\
\hline Control & $78 \pm 6 \mathrm{~d}$ & $80 \pm 6 \mathrm{~d}$ & $25.3 \pm 2.9 \mathrm{~d}$ & $26.0 \pm 3.0 \mathrm{~d}$ & $2.83 \pm 0.17 \mathrm{~d}$ & $2.92 \pm 0.18 \mathrm{~d}$ \\
\hline 10 & $88 \pm 12 c$ & $91 \pm 12 c$ & $33.5 \pm 9.0 c$ & $34.5 \pm 9.3 c$ & $3.19 \pm 0.45 c$ & $3.29 \pm 0.47 c$ \\
\hline 20 & $97 \pm 9 \mathrm{~b}$ & $100 \pm 9 b$ & $37.5 \pm 6.7 b$ & $38.6 \pm 6.9 \mathrm{~b}$ & $3.55 \pm 0.51 b$ & $3.66 \pm 0.52 b$ \\
\hline 30 & $105 \pm 10 \mathrm{a}$ & $108 \pm 10$ & $41.5 \pm 6.9 \mathrm{a}$ & $42.7 \pm 7.1 \mathrm{a}$ & $4.02 \pm 0.68 \mathrm{a}$ & $4.13 \pm 0.70 \mathrm{a}$ \\
\hline \multicolumn{7}{|c|}{ Combination treatments } \\
\hline Control & $72 \pm 1 \mathrm{~h}$ & $74 \pm 1 \mathrm{~h}$ & $22.7 \pm 0.2 \mathrm{~h}$ & $23.3 \pm 0.2 \mathrm{~h}$ & $2.68 \pm 0.03 \mathrm{~h}$ & $2.76 \pm 0.03 \mathrm{~h}$ \\
\hline $\mathrm{T} 1$ & $77 \pm 1 \mathrm{~g}$ & $80 \pm 1 \mathrm{~g}$ & $25.2 \pm 0.2 \mathrm{~g}$ & $26.0 \pm 0.2 \mathrm{~g}$ & $2.78 \pm 0.03 \mathrm{~g}$ & $2.86 \pm 0.03 \mathrm{~g}$ \\
\hline $\mathrm{T} 2$ & $90 \pm 1 \mathrm{e}$ & $92 \pm 1 \mathrm{e}$ & $31.4 \pm 0.3 \mathrm{e}$ & $32.3 \pm 0.3 \mathrm{e}$ & $3.09 \pm 0.03 \mathrm{e}$ & $3.18 \pm 0.03 \mathrm{e}^{\circ}$ \\
\hline T3 & $96 \pm 1 \mathrm{~d}$ & $99 \pm 1 \mathrm{~d}$ & $35.2 \pm 0.3 \mathrm{~d}$ & $36.3 \pm 0.3 \mathrm{~d}$ & $3.40 \pm 0.03 \mathrm{~d}$ & $3.50 \pm 0.03 \mathrm{~d}$ \\
\hline $\mathrm{T} 4$ & $83 \pm 1 \mathrm{f}$ & $86 \pm 1 \mathrm{f}$ & $27.9 \pm 0.3 \mathrm{f}$ & $28.7 \pm 0.3 \mathrm{f}$ & $2.99 \pm 0.03 \mathrm{f}$ & $3.07 \pm 0.03 \mathrm{f}$ \\
\hline $\mathrm{T} 5$ & $99 \pm 1 \mathrm{c}$ & $102 \pm 1 c$ & $41.7 \pm 0.4 \mathrm{c}$ & $42.9 \pm 0.4 c$ & $3.61 \pm 0.04 c$ & $3.71 \pm 0.04 \mathrm{c}$ \\
\hline T6 & $105 \pm 1 b$ & $108 \pm 1 b$ & $43.6 \pm 0.4 b$ & $44.8 \pm 0.4 b$ & $4.02 \pm 0.04 b$ & $4.13 \pm 0.04 b$ \\
\hline $\mathrm{T} 7$ & $113 \pm 1 \mathrm{a}$ & $117 \pm 1 \mathrm{a}$ & $47.8 \pm 0.5 \mathrm{a}$ & $49.2 \pm 0.5 \mathrm{a}$ & $4.64 \pm 0.05 \mathrm{a}$ & $4.77 \pm 0.05 \mathrm{a}$ \\
\hline
\end{tabular}

Data are mean value \pm SE. Means in columns followed by the same letter are not statistically different at the 0.05 significance level.

Table 6. The mean values of the number of secondary branches, full bloom date (day) and flowering percentage (\%) of jojoba shrubs affected with ground inoculation of arbuscular mycorrhizal fungi and foliar application of Moringa oleifera leaves extract, as well as their combination treatments in both seasons of the study.

\begin{tabular}{|c|c|c|c|c|c|c|}
\hline \multirow{2}{*}{ Treatment } & \multicolumn{2}{|c|}{ Number of Secondary Branches } & \multicolumn{2}{|c|}{ Full Bloom Date (Day) } & \multicolumn{2}{|c|}{ Flowering Percentage (\%) } \\
\hline & 1st Season & 2nd Season & 1st Season & 2nd Season & 1st Season & 2nd Season \\
\hline \multicolumn{7}{|c|}{ Arbuscular mycorrhizal fungi $\left(\mathrm{g} \mathrm{L}^{-1}\right)$} \\
\hline Control & $4.38 \pm 0.63 b$ & $4.51 \pm 0.65 b$ & $56.9 \pm 4.3 \mathrm{a}$ & $58.6 \pm 4.4 \mathrm{a}$ & $46.35 \pm 4.11 \mathrm{~b}$ & $47.70 \pm 4.23 \mathrm{~b}$ \\
\hline 20 & $5.94 \pm 1.05 \mathrm{a}$ & $6.11 \pm 1.08 \mathrm{a}$ & $49.4 \pm 5.3 b$ & $50.9 \pm 5.5 b$ & $53.30 \pm 4.48 \mathrm{a}$ & $54.86 \pm 4.61 \mathrm{a}$ \\
\hline \multicolumn{7}{|c|}{ Moringa oleifera leaves extract $\left(\mathrm{g} \mathrm{L}^{-1}\right)$} \\
\hline Control & $3.97 \pm 0.40 \mathrm{~d}$ & $4.08 \pm 0.41 \mathrm{~d}$ & $59.7 \pm 2.3 \mathrm{a}$ & $61.48 \pm 2.4 \mathrm{a}$ & $43.78 \pm 2.85 \mathrm{~d}$ & $45.05 \pm 2.93 \mathrm{~d}$ \\
\hline 10 & $4.94 \pm 1.02 \mathrm{c}$ & $5.09 \pm 1.05 c$ & $54.6 \pm 5.7 \mathrm{~b}$ & $56.18 \pm 5.8 b$ & $48.93 \pm 5.10 \mathrm{c}$ & $50.35 \pm 5.24 c$ \\
\hline 20 & $5.67 \pm 1.02 b$ & $5.83 \pm 1.05 b$ & $50.5 \pm 4.5 c$ & $51.94 \pm 4.7 \mathrm{c}$ & $52.02 \pm 3.97 b$ & $53.53 \pm 4.09 \mathrm{~b}$ \\
\hline 30 & $6.06 \pm 0.98 \mathrm{a}$ & $6.23 \pm 1.01 \mathrm{a}$ & $47.9 \pm 4.0 \mathrm{~d}$ & $49.29 \pm 4.1 \mathrm{~d}$ & $54.59 \pm 3.42 \mathrm{a}$ & $56.18 \pm 3.52 \mathrm{a}$ \\
\hline \multicolumn{7}{|c|}{ Combination treatments } \\
\hline (Control) & $3.61 \pm 0.04 \mathrm{~h}$ & $3.71 \pm 0.04 \mathrm{~h}$ & $61.8 \pm 0.6 \mathrm{a}$ & $63.6 \pm 0.6 \mathrm{a}$ & $41.20 \pm 0.40 \mathrm{~h}$ & $42.40 \pm 0.40 \mathrm{~h}$ \\
\hline $\mathrm{T} 1$ & $4.02 \pm 0.04 \mathrm{~g}$ & $4.13 \pm 0.04 \mathrm{~g}$ & $59.7 \pm 0.6 b$ & $61.5 \pm 0.6 b$ & $44.29 \pm 0.43 \mathrm{~g}$ & $45.58 \pm 0.43 \mathrm{~g}$ \\
\hline $\mathrm{T} 2$ & $4.74 \pm 0.04 \mathrm{e}$ & $4.88 \pm 0.05 \mathrm{e}$ & $54.6 \pm 0.5 \mathrm{~d}$ & $56.2 \pm 0.5 \mathrm{~d}$ & $48.41 \pm 0.47 \mathrm{e}$ & $49.82 \pm 0.47 \mathrm{e}$ \\
\hline T3 & $5.16 \pm 0.04 \mathrm{~d}$ & $5.31 \pm 0.05 \mathrm{~d}$ & $51.5 \pm 0.5 \mathrm{e}$ & $53.0 \pm 0.5 \mathrm{e}$ & $51.5 \pm 0.50 \mathrm{~d}$ & $53.00 \pm 0.50 \mathrm{~d}$ \\
\hline $\mathrm{T} 4$ & $4.33 \pm 0.04 \mathrm{f}$ & $4.45 \pm 0.04 \mathrm{f}$ & $57.7 \pm 0.6 \mathrm{c}$ & $59.4 \pm 0.6 c$ & $46.35 \pm 0.45 \mathrm{f}$ & $47.70 \pm 0.45 \mathrm{f}$ \\
\hline $\mathrm{T} 5$ & $5.87 \pm 0.04 c$ & $6.04 \pm 0.06 c$ & $49.4 \pm 0.5 \mathrm{f}$ & $50.9 \pm 0.5 \mathrm{f}$ & $53.56 \pm 0.52 c$ & $55.12 \pm 0.52 c$ \\
\hline T6 & $6.59 \pm 0.04 b$ & $6.78 \pm 0.06 b$ & $46.4 \pm 0.5 \mathrm{~g}$ & $47.7 \pm 0.5 \mathrm{~g}$ & $55.62 \pm 0.54 b$ & $57.24 \pm 0.54 b$ \\
\hline $\mathrm{T} 7$ & $6.95 \pm 0.07 \mathrm{a}$ & $7.16 \pm 0.07 a$ & $44.3 \pm 0.4 \mathrm{~h}$ & $45.6 \pm 0.4 \mathrm{~h}$ & $57.68 \pm 0.56 a$ & $59.36 \pm 0.56 a$ \\
\hline
\end{tabular}

Data are mean value \pm SE. Means in columns followed by the same letter are not statistically different at the 0.05 significance level. 


\subsection{Flowering and Fruits Set Parameters}

Data shown in Tables 6 and 7 are clear that foliar application of ground inoculation of arbuscular mycorrhizal fungi and Moringa oleifera leaves extract, as well as their combined treatments, significantly promoted all flowering and fruits set parameters compared with untreated control treatment. When using mycorrhizal fungi, the reduction in full bloom date was $13 \%$ while the increment in flowering and final fruit set percent was 15 and $4 \%$, respectively, compared with untreated shrubs. Flowering and fruits set parameters were significantly improved using $30 \mathrm{~g} \mathrm{~L}^{-1}$ Moringa oleifera leaves extract compared with the other extract concentrations and control. The reduction in full bloom date was $20 \%$ and the increment in flowering and final fruit set percent was 25 and 7\%, respectively, compared with control shrubs. Regarding the combined treatments, the most significant increases in flowering and fruits set parameters were obtained at the combination treatment of arbuscular mycorrhizal fungi $\left(20 \mathrm{~g} \mathrm{~L}^{-1}\right)$ plus $30 \mathrm{~g} \mathrm{~L}^{-1}$ Moringa oleifera leaves extract in both seasons. Moreover, it achieved a reduction percent in full bloom date with $20 \%$, while it achieved an increment in flowering and final fruit set percent with 25 and $11 \%$, respectively.

Table 7. The mean values of the final fruit set $(\%)$, seed yield $\left(\mathrm{g} \mathrm{plant}^{-1}\right)$ and seed yield $\left(\mathrm{Kg} \mathrm{ha}^{-1}\right)$ of jojoba shrubs as affected with ground inoculation of arbuscular mycorrhizal fungi and foliar application of Moringa oleifera leaves extract, as well as their combination treatments in both seasons of the study.

\begin{tabular}{|c|c|c|c|c|c|c|}
\hline \multirow{2}{*}{ Treatment } & \multicolumn{2}{|c|}{ Final Fruit Set (\%) } & \multicolumn{2}{|c|}{ Seed Yield (g plant ${ }^{-1}$ ) } & \multicolumn{2}{|c|}{ Seed Yield (Kg ha $\left.{ }^{-1}\right)$} \\
\hline & 1st Season & 2nd Season & 1st Season & 2nd Season & 1st Season & 2nd Season \\
\hline \multicolumn{7}{|c|}{ Arbuscular mycorrhizal fungi $\left(\mathrm{g} \mathrm{L}^{-1}\right)$} \\
\hline Control & $86.06 \pm 1.89 b$ & $88.56 \pm 1.94 b$ & $2198 \pm 180 \mathrm{~b}$ & $2262 \pm 185 b$ & $2931 \pm 240 b$ & $3016 \pm 247 b$ \\
\hline 20 & $89.56 \pm 2.80 \mathrm{a}$ & $92.17 \pm 2.88 \mathrm{a}$ & $2477 \pm 176 \mathrm{a}$ & $2549 \pm 181 \mathrm{a}$ & $3303 \pm 235 a$ & $3399 \pm 241 \mathrm{a}$ \\
\hline \multicolumn{7}{|c|}{ Moringa oleifera leaves extract $\left(\mathrm{g} \mathrm{L}^{-1}\right)$} \\
\hline Control & $84.87 \pm 1.35 \mathrm{~d}$ & $87.34 \pm 1.38 \mathrm{~d}$ & $2088 \pm 134 d$ & $2149 \pm 138 d$ & $2784 \pm 178 \mathrm{~d}$ & $2866 \pm 184 \mathrm{~d}$ \\
\hline 10 & $87.04 \pm 2.27 c$ & $89.57 \pm 2.33 c$ & $2294 \pm 201 c$ & $2361 \pm 207 c$ & $3059 \pm 268 c$ & $3148 \pm 276 c$ \\
\hline 20 & $88.73 \pm 1.81 b$ & $91.32 \pm 1.85 b$ & $2434 \pm 145 b$ & $2505 \pm 149 b$ & $3245 \pm 193 b$ & $3340 \pm 199 b$ \\
\hline 30 & $90.59 \pm 2.87 \mathrm{a}$ & $93.23 \pm 2.95 \mathrm{a}$ & $2534 \pm 137 \mathrm{a}$ & $2608 \pm 140 \mathrm{a}$ & $3379 \pm 182 \mathrm{a}$ & $3478 \pm 187 a$ \\
\hline \multicolumn{7}{|c|}{ Combination treatments } \\
\hline Control & $83.84 \pm 0.81 \mathrm{~h}$ & $86.28 \pm 0.81 \mathrm{~h}$ & $1967 \pm 19 \mathrm{~h}$ & $2025 \pm 19 \mathrm{~h}$ & $2623 \pm 25 \mathrm{~h}$ & $2699 \pm 25 \mathrm{~h}$ \\
\hline $\mathrm{T} 1$ & $85.08 \pm 0.83 \mathrm{~g}$ & $87.56 \pm 0.83 \mathrm{~g}$ & $2112 \pm 21 \mathrm{~g}$ & $2173 \pm 21 \mathrm{~g}$ & $2815 \pm 27 \mathrm{~g}$ & $2897 \pm 27 \mathrm{~g}$ \\
\hline $\mathrm{T} 2$ & $87.24 \pm 0.85 \mathrm{e}$ & $89.78 \pm 0.85 \mathrm{e}$ & $2303 \pm 22 \mathrm{e}$ & $2370 \pm 22 \mathrm{e}$ & $3071 \pm 30 \mathrm{e}$ & $3160 \pm 30 \mathrm{e}$ \\
\hline T3 & $88.07 \pm 0.86 \mathrm{~d}$ & $90.63 \pm 0.86 \mathrm{~d}$ & $2411 \pm 23 \mathrm{~d}$ & $2481 \pm 23 \mathrm{~d}$ & $3215 \pm 31 \mathrm{~d}$ & $3309 \pm 31 d$ \\
\hline $\mathrm{T} 4$ & $85.90 \pm 0.83 \mathrm{f}$ & $88.40 \pm 0.83 \mathrm{f}$ & $2209 \pm 21 \mathrm{f}$ & $2274 \pm 21 \mathrm{f}$ & $2946 \pm 29 \mathrm{f}$ & $3032 \pm 29 \mathrm{f}$ \\
\hline $\mathrm{T} 5$ & $88.99 \pm 0.86 c$ & $91.58 \pm 0.86 c$ & $2477 \pm 24 c$ & $2549 \pm 24 c$ & $3303 \pm 32 c$ & $3399 \pm 32 c$ \\
\hline T6 & $90.23 \pm 0.88 b$ & $92.86 \pm 0.88 b$ & $2565 \pm 25 b$ & $2639 \pm 25 b$ & $3420 \pm 33 b$ & $3519 \pm 33 b$ \\
\hline $\mathrm{T} 7$ & $93.11 \pm 0.90 \mathrm{a}$ & $95.82 \pm 0.90 \mathrm{a}$ & $2657 \pm 26 a$ & $2735 \pm 26 a$ & $3543 \pm 34 a$ & $3646 \pm 34 a$ \\
\hline
\end{tabular}

Data are mean value \pm SE. Means in columns followed by the same letter are not statistically different at the 0.05 significance level.

\subsection{Seeds Yield}

Seeds yield per tree and per hectare of jojoba shrubs have been significantly affected with the application of both ground inoculation of arbuscular mycorrhizal fungi and Moringa oleifera leaves extract (Table 7). Therefore, using arbuscular mycorrhizal fungi had a $13 \%$ increment in seed yield per tree and per hectare of jojoba shrubs, respectively, in both seasons compared with the control. Additionally, foliar application of $30 \mathrm{~g} \mathrm{~L}^{-1}$ gave a maximum increment in seed yield per tree $(21 \%)$ and per hectare $(21 \%)$ compared with control. Likewise for the combined effect, the treatment of arbuscular mycorrhizal fungi $\left(20 \mathrm{~g} \mathrm{~L}^{-1}\right)$ plus $30 \mathrm{~g} \mathrm{~L}^{-1}$ Moringa oleifera leaves extract recorded the maximum significant seed yield per tree and per hectare in both seasons. In addition, it recorded the maximum increment percentage compared with the control treatment for the yield of seeds per tree $(35 \%)$ as well as per hectare $(35 \%)$ in both seasons, respectively. The most pronounced 
effect was recorded with the application of ground inoculation of arbuscular mycorrhizal fungi and $30 \mathrm{~g} \mathrm{~L}^{-1}$ Moringa oleifera leaves extract for combination treatment.

\subsection{Seeds Chemical Compounds}

Tables 8 and 9 show the effect of arbuscular mycorrhizal fungi and Moringa oleifera leaves extract rates on chemical seed compounds of jojoba shrubs. Arbuscular mycorrhizal fungi application caused significant maximum increases in minerals, proteins and fixed oil percent with a significant decrease in carbohydrate percentage. Amendment of soil with arbuscular mycorrhizal fungi increased the percent of minerals, proteins and fixed oil percent by $17 \%, 9 \%$ and $2 \%$ compared with the inoculated plants. The maximum carbohydrate percentage was $27.28 \%$ in the first season in the control and $25.16 \%$ in the second season. For the application of Moringa oleifera leaves extract, foliar application of $30 \mathrm{~g} \mathrm{~L}^{-1}$ had significant maximum increases in minerals, proteins and fixed oil percentage by $25 \%, 14 \%$ and $8 \%$, while the control shrubs recorded the maximum carbohydrate percentage in the jojoba seeds (25.61 and $23.45 \%$ ) in the first and second seasons, respectively. Regarding the combination treatments, all treatments improved minerals, proteins and fixed oil percent in jojoba seeds in both seasons compared with the control. In addition, the treatment of arbuscular mycorrhizal fungi $\left(20 \mathrm{~g} \mathrm{~L}^{-1}\right)$ plus $30 \mathrm{~g} \mathrm{~L}^{-1}$ Moringa oleifera leaves extract gave the significant maximum increases in mineral and protein percentages, as it increased the percent of minerals and proteins percentage by $42 \%$ and $23 \%$, respectively, above the control in both seasons. For the fixed oil percentage, the treatment of uninoculated soil plus $30 \mathrm{~g} \mathrm{~L}^{-1}$ Moringa oleifera leaves extract had the maximum percent which was above the control treatment with $14 \%$ in both seasons. On the contrary, the maximum carbohydrate percentage (27.28 and $25.16 \%$ ) in both seasons, respectively, was found with the shrubs of the control treatment.

Table 8. The mean values of minerals (\%), proteins (\%) and carbohydrates percentage (\%) of jojoba shrubs affected with ground inoculation of arbuscular mycorrhizal fungi and foliar application of Moringa oleifera leaves extract, as well as their combination treatments in both seasons of the study.

\begin{tabular}{|c|c|c|c|c|c|c|}
\hline \multirow{2}{*}{ Treatment } & \multicolumn{2}{|c|}{ Minerals (\%) } & \multicolumn{2}{|c|}{ Proteins (\%) } & \multicolumn{2}{|c|}{ Carbohydrates (\%) } \\
\hline & 1st Season & 2nd Season & 1st Season & 2nd Season & 1st Season & 2nd Season \\
\hline \multicolumn{7}{|c|}{ Arbuscular mycorrhizal fungi $\left(\mathrm{g} \mathrm{L}^{-1}\right)$} \\
\hline Control & $1.22 \pm 0.08 \mathrm{~b}$ & $1.26 \pm 0.08 \mathrm{~b}$ & $23.14 \pm 1.11 b$ & $23.81 \pm 1.15 b$ & $23.16 \pm 3.94 \mathrm{a}$ & $20.92 \pm 4.05 \mathrm{a}$ \\
\hline 20 & $1.43 \pm 0.14 \mathrm{a}$ & $1.47 \pm 0.14 \mathrm{a}$ & $25.30 \pm 1.54 \mathrm{a}$ & $26.04 \pm 1.58 \mathrm{a}$ & $19.93 \pm 2.54 b$ & $17.60 \pm 2.61 b$ \\
\hline \multicolumn{7}{|c|}{ Moringa oleifera leaves extract $\left(\mathrm{g} \mathrm{L}^{-1}\right)$} \\
\hline Control & $1.18 \pm 0.05 \mathrm{~d}$ & $1.21 \pm 0.05 \mathrm{~d}$ & $22.27 \pm 0.73 \mathrm{~d}$ & $22.92 \pm 0.75 \mathrm{~d}$ & $25.61 \pm 1.94 \mathrm{a}$ & $23.45 \pm 1.99 \mathrm{a}$ \\
\hline 10 & $1.30 \pm 0.14 c$ & $1.34 \pm 0.14 c$ & $24.01 \pm 1.47 \mathrm{c}$ & $24.71 \pm 1.51 \mathrm{c}$ & $21.95 \pm 3.91 b$ & $19.68 \pm 4.02 b$ \\
\hline 20 & $1.36 \pm 0.12 b$ & $1.40 \pm 0.13 b$ & $25.15 \pm 1.25 \mathrm{~b}$ & $25.88 \pm 1.29 \mathrm{~b}$ & $20.28 \pm 2.44 c$ & $17.96 \pm 2.50 c$ \\
\hline 30 & $1.47 \pm 0.14 \mathrm{a}$ & $1.51 \pm 0.15 \mathrm{a}$ & $25.45 \pm 1.37 \mathrm{a}$ & $26.19 \pm 1.41 \mathrm{a}$ & $18.33 \pm 1.18 \mathrm{~d}$ & $15.95 \pm 1.21 \mathrm{~d}$ \\
\hline \multicolumn{7}{|c|}{ Combination treatments } \\
\hline Control & $1.13 \pm 0.01 \mathrm{~h}$ & $1.17 \pm 0.01 \mathrm{~h}$ & $21.63 \pm 0.21 \mathrm{~h}$ & $22.26 \pm 0.21 \mathrm{~h}$ & $27.28 \pm 0.71 \mathrm{a}$ & $25.16 \pm 0.71 \mathrm{a}$ \\
\hline $\mathrm{T} 1$ & $1.17 \pm 0.01 \mathrm{~g}$ & $1.21 \pm 0.01 \mathrm{~g}$ & $22.68 \pm 0.22 \mathrm{~g}$ & $23.34 \pm 0.22 \mathrm{~g}$ & $25.47 \pm 0.72 b$ & $23.30 \pm 0.72 b$ \\
\hline $\mathrm{T} 2$ & $1.25 \pm 0.01 \mathrm{e}$ & $1.28 \pm 0.01 \mathrm{e}$ & $24.02 \pm 0.23 \mathrm{e}$ & $24.72 \pm 0.23 \mathrm{e}$ & $22.41 \pm 0.75 \mathrm{~d}$ & $20.15 \pm 0.75 \mathrm{~d}$ \\
\hline T3 & $1.34 \pm 0.01 \mathrm{~d}$ & $1.38 \pm 0.01 \mathrm{~d}$ & $24.22 \pm 0.24 \mathrm{~d}$ & $24.92 \pm 0.24 \mathrm{~d}$ & $17.47 \pm 0.80 \mathrm{~h}$ & $15.06 \pm 0.80 \mathrm{~h}$ \\
\hline $\mathrm{T} 4$ & $1.23 \pm 0.01 \mathrm{f}$ & $1.26 \pm 0.01 \mathrm{f}$ & $22.92 \pm 0.22 \mathrm{f}$ & $23.59 \pm 0.22 \mathrm{f}$ & $23.94 \pm 0.74 \mathrm{c}$ & $21.73 \pm 0.74 c$ \\
\hline $\mathrm{T} 5$ & $1.42 \pm 0.01 \mathrm{c}$ & $1.46 \pm 0.01 c$ & $25.34 \pm 0.25 c$ & $26.08 \pm 0.25 c$ & $18.43 \pm 0.79 \mathrm{f}$ & $16.06 \pm 0.79 \mathrm{f}$ \\
\hline T6 & $1.47 \pm 0.01 \mathrm{~b}$ & $1.52 \pm 0.01 b$ & $26.28 \pm 0.26 b$ & $27.04 \pm 0.26 b$ & $18.15 \pm 0.79 \mathrm{~g}$ & $15.76 \pm 0.79 \mathrm{~g}$ \\
\hline $\mathrm{T} 7$ & $1.60 \pm 0.02 \mathrm{a}$ & $1.64 \pm 0.02 \mathrm{a}$ & $26.68 \pm 0.26 a$ & $27.45 \pm 0.26 \mathrm{a}$ & $19.20 \pm 0.78 \mathrm{e}$ & $16.84 \pm 0.78 \mathrm{e}$ \\
\hline
\end{tabular}

Data are mean value \pm SE. Means in columns followed by the same letter are not statistically different at the 0.05 significance level. 
Table 9. The mean values of oil percent (\%), oil content $\left(\mathrm{mL} \mathrm{plant}^{-1}\right)$ and oil yield $\left(1 \mathrm{ha}^{-1}\right)$ of jojoba shrub affected with ground inoculation of arbuscular mycorrhizal fungi and foliar application of Moringa oleifera leaves extract, as well as their combination treatments in both seasons of the study.

\begin{tabular}{|c|c|c|c|c|c|c|}
\hline \multirow{2}{*}{ Treatment } & \multicolumn{2}{|c|}{ Oil Percent (\%) } & \multicolumn{2}{|c|}{ Oil Content (mL plant ${ }^{-1}$ ) } & \multicolumn{2}{|c|}{ Oil Yield (1 ha $\left.{ }^{-1}\right)$} \\
\hline & 1st Season & 2nd Season & 1st Season & 2nd Season & 1st Season & 2nd Season \\
\hline \multicolumn{7}{|c|}{ Arbuscular mycorrhizal fungi $\left(\mathrm{g} \mathrm{L}^{-1}\right)$} \\
\hline Control & $52.48 \pm 2.89 \mathrm{~b}$ & $54.01 \pm 2.97 b$ & $1158 \pm 155 b$ & $1226 \pm 165 b$ & $1544 \pm 207 b$ & $1635 \pm 219 b$ \\
\hline 20 & $53.34 \pm 1.29 \mathrm{a}$ & $54.89 \pm 1.33 \mathrm{a}$ & $1322 \pm 109 \mathrm{a}$ & $1400 \pm 115 \mathrm{a}$ & $1763 \pm 145 \mathrm{a}$ & $1867 \pm 154 \mathrm{a}$ \\
\hline \multicolumn{7}{|c|}{ Moringa oleifera leaves extract $\left(\mathrm{g} \mathrm{L}^{-1}\right)$} \\
\hline Control & $50.93 \pm 1.16 \mathrm{~d}$ & $52.42 \pm 1.19 \mathrm{~d}$ & $1065 \pm 92 \mathrm{~d}$ & $1128 \pm 97 \mathrm{~d}$ & $1420 \pm 122 \mathrm{~d}$ & $1504 \pm 129 \mathrm{~d}$ \\
\hline 10 & $52.74 \pm 2.31 c$ & $54.28 \pm 2.37 c$ & $1214 \pm 159 c$ & $1286 \pm 168 c$ & $1619 \pm 212 c$ & $1714 \pm 224 \mathrm{c}$ \\
\hline 20 & $53.21 \pm 1.08 \mathrm{~b}$ & $54.76 \pm 1.11 \mathrm{~b}$ & $1296 \pm 103 b$ & $1373 \pm 108 b$ & $1729 \pm 137 b$ & $1831 \pm 145 b$ \\
\hline 30 & $54.75 \pm 2.48 \mathrm{a}$ & $56.35 \pm 2.55 \mathrm{a}$ & $1385 \pm 27 \mathrm{a}$ & $1467 \pm 28 \mathrm{a}$ & $1847 \pm 36 \mathrm{a}$ & $1956 \pm 37 \mathrm{a}$ \\
\hline \multicolumn{7}{|c|}{ Combination treatments } \\
\hline Control & $49.96 \pm 0.49 h$ & $51.41 \pm 0.49 \mathrm{~h}$ & $983 \pm 19 h$ & $1041 \pm 20 \mathrm{~h}$ & $1310 \pm 25 \mathrm{~h}$ & $1388 \pm 26 h$ \\
\hline $\mathrm{T} 1$ & $50.68 \pm 0.49 \mathrm{~g}$ & $52.15 \pm 0.49 \mathrm{~g}$ & $1070 \pm 21 \mathrm{~g}$ & $1133 \pm 21 \mathrm{~g}$ & $1427 \pm 28 \mathrm{~g}$ & $1511 \pm 29 \mathrm{~g}$ \\
\hline $\mathrm{T} 2$ & $52.32 \pm 0.51 \mathrm{e}$ & $53.85 \pm 0.51 \mathrm{e}$ & $1205 \pm 23 \mathrm{e}$ & $1276 \pm 24 \mathrm{e}$ & $1607 \pm 31 \mathrm{e}$ & $1702 \pm 32 \mathrm{e}$ \\
\hline $\mathrm{T} 3$ & $56.98 \pm 0.55 a$ & $58.64 \pm 0.55 a$ & $1374 \pm 27 \mathrm{c}$ & $1455 \pm 27 c$ & $1832 \pm 36 c$ & $1940 \pm 37 c$ \\
\hline $\mathrm{T} 4$ & $51.91 \pm 0.50 \mathrm{f}$ & $53.42 \pm 0.50 \mathrm{f}$ & $1147 \pm 22 \mathrm{f}$ & $1215 \pm 23 \mathrm{f}$ & $1529 \pm 30 \mathrm{f}$ & $1620 \pm 31 \mathrm{f}$ \\
\hline $\mathrm{T} 5$ & $54.81 \pm 0.53 b$ & $56.40 \pm 0.53 b$ & $1358 \pm 26 \mathrm{~d}$ & $1438 \pm 27 \mathrm{~d}$ & $1810 \pm 35 \mathrm{~d}$ & $1917 \pm 36 \mathrm{~d}$ \\
\hline T6 & $54.11 \pm 0.53 c$ & $55.68 \pm 0.53 c$ & $1388 \pm 27 b$ & $1470 \pm 28 b$ & $1850 \pm 36 b$ & $1960 \pm 37 b$ \\
\hline $\mathrm{T} 7$ & $52.53 \pm 0.51 \mathrm{~d}$ & $54.06 \pm 0.51 \mathrm{~d}$ & $1396 \pm 27 a$ & $1479 \pm 28 \mathrm{a}$ & $1861 \pm 36 \mathrm{a}$ & $1971 \pm 37 a$ \\
\hline
\end{tabular}

Data are mean value \pm SE. Means in columns followed by the same letter are not statistically different at the 0.05 significance level.

\subsection{Yield of Seeds Oil}

Table 9 shows the effect of arbuscular mycorrhizal fungi and Moringa oleifera leaves extract on jojoba seeds oil content per shrub and yield per hectare. Amendment of soil with arbuscular mycorrhizal fungi caused significant increases in jojoba seed oil content per shrub and yield per hectare. The yield of jojoba seeds oil content per shrub and yield per hectare ranged between 1158 and $1322 \mathrm{~mL} \mathrm{shrub}^{-1}$ and 1763 and $1544 \mathrm{~L} \mathrm{ha}^{-1}$ in the first season and between 1226 to $1400 \mathrm{~mL} \mathrm{shrub}^{-1}$ and 1635 to $1867 \mathrm{~L} \mathrm{ha}^{-1}$ in the second season. The highest significant values of jojoba seed oil content per shrub and yield per hectare were noticed with the soil amended with arbuscular mycorrhizal fungi, while the lowest values were found with the control soil. Significantly, the application of Moringa oleifera leaves extract with $30 \mathrm{~g} \mathrm{~L}^{-1}$ had the maximum jojoba seed oil content per shrub and yield per hectare, as it increased the seeds' oil content per shrub and yield per hectare by $30 \%$ and $30 \%$, respectively, compared with non-foliar shrubs. For the combination treatments, arbuscular mycorrhizal fungi $\left(20 \mathrm{~g} \mathrm{~L}^{-1}\right)$ plus $30 \mathrm{~g} \mathrm{~L}^{-1}$ Moringa oleifera leaves extract gave the maximum significant jojoba seed oil content per shrub and yield per hectare, as it increased seed oil content per shrub and yield per hectare by $42 \%$ and $42 \%$, respectively, above untreated shrubs.

\subsection{Seeds Fixed Oil Analysis}

It was noticed from Table 10 that the fixed oil of jojoba seeds is rich with unsaturated fatty acids, which ranged between 87.99 and $95.88 \%$ for $\mathrm{T} 7$ and control treatment. The total known components ranged between 91.82 to $97.94 \%$ for T7 and control, respectively. The major fatty acids in jojoba oil are Gadoleic acid (48.21-52.29\% for T3 and T1), Oleic acid (12.03-14.58\% for T6 and control), Erucic acid (12.68-15.28\% for T7 and T4) and Nervonic acid (11.7-12.78\% for T2 and T1). 
Table 10. The relative percentage of fatty acids (\%) of fixed oil of Jojoba oil affected with combination treatments of ground inoculation of Arbuscular mycorrhizal fungi and foliar application of Moringa oleifera leaves extract.

\begin{tabular}{|c|c|c|c|c|c|c|c|c|}
\hline \multirow{2}{*}{ Fatty Acids } & \multicolumn{8}{|c|}{ The Relative Percentage of Fatty Acids (\%) } \\
\hline & Control & T1 & T2 & T3 & T4 & T5 & T6 & T7 \\
\hline $\begin{array}{l}\text { Myristic Acid } \\
\text { (C14:0) }\end{array}$ & $0.77 \pm 0.01$ & $0.52 \pm 0.01$ & $1.05 \pm 0.01$ & - & $0.89 \pm 0.01$ & $1.55 \pm 0.02$ & $1.77 \pm 0.02$ & $2.09 \pm 0.02$ \\
\hline $\begin{array}{c}\text { Myristoleic Acid } \\
\text { (C14:1) }\end{array}$ & $1.4 \pm 0.01$ & - & $1.23 \pm 0.01$ & $1.13 \pm 0.01$ & - & - & $0.67 \pm 0.01$ & $0.35 \pm 0.00$ \\
\hline $\begin{array}{c}\text { PalmiticÁcic } \\
(\mathrm{C} 16: 0)\end{array}$ & $1.29 \pm 0.01$ & $1.17 \pm 0.01$ & $1.43 \pm 0.01$ & $1.56 \pm 0.02$ & $1.33 \pm 0.01$ & $1.61 \pm 0.02$ & $1.69 \pm 0.02$ & $1.86 \pm 0.02$ \\
\hline $\begin{array}{l}\text { Oleic acid } \\
\text { (C18:1) }\end{array}$ & $14.58 \pm 0.18$ & $14.44 \pm 0.17$ & $13.52 \pm 0.16$ & $13.05 \pm 0.15$ & $13.93 \pm 0.16$ & $12.68 \pm 0.15$ & $12.03 \pm 0.14$ & $12.53 \pm 0.14$ \\
\hline $\begin{array}{l}\text { Linoleic acid } \\
(\mathrm{C} 18: 2)\end{array}$ & $2.17 \pm 0.02$ & $2.25 \pm 0.02$ & $1.96 \pm 0.02$ & $1.94 \pm 0.02$ & $2.09 \pm 0.02$ & $1.72 \pm 0.02$ & $1.45 \pm 0.01$ & $1.32 \pm 0.01$ \\
\hline $\begin{array}{l}\text { Gadoleic acid } \\
\text { (C20:1) }\end{array}$ & $51.84 \pm 0.30$ & $52.29 \pm 0.58$ & $49.49 \pm 0.56$ & $48.21 \pm 0.54$ & $50.96 \pm 0.57$ & $48.64 \pm 0.53$ & $49.56 \pm 0.52$ & $48.58 \pm 0.51$ \\
\hline $\begin{array}{l}\text { Erucic acid } \\
(\mathrm{C} 22: 1)\end{array}$ & $13.7 \pm 0.14$ & $14.03 \pm 0.14$ & $13.25 \pm 0.14$ & $14.62 \pm 0.13$ & $15.28 \pm 0.14$ & $13.99 \pm 0.12$ & $13.23 \pm 0.12$ & $12.68 \pm 0.11$ \\
\hline $\begin{array}{l}\text { Nervonic acid } \\
\text { (C24:1) }\end{array}$ & $12.19 \pm 0.20$ & $12.78 \pm 0.19$ & $11.7 \pm 0.18$ & $11.31 \pm 0.18$ & $12.10 \pm 0.19$ & $12.91 \pm 0.17$ & $12.42 \pm 0.16$ & $12.53 \pm 0.15$ \\
\hline Total \% & 97.94 & 97.48 & 93.63 & 91.82 & 96.58 & 93.1 & 92.82 & 91.94 \\
\hline Saturated fatty acids & 2.06 & 1.69 & 2.48 & 1.56 & 2.22 & 3.16 & 3.46 & 3.95 \\
\hline Unsaturated fatty acids & 95.88 & 95.79 & 91.15 & 90.26 & 94.36 & 89.94 & 89.36 & 87.99 \\
\hline
\end{tabular}

\subsection{Chlorophyll $a$ and $b$}

All treatments of arbuscular mycorrhizal fungi and Moringa oleifera leaves extract and their interaction recorded significant increases on chlorophyll a and $b$ of jojoba leaves in both seasons (Table 11). Amendment of soil with arbuscular mycorrhizal fungi caused remarkable increases in chlorophyll $\mathrm{a}$ and $\mathrm{b}$ (Table 11). Chlorophyll $\mathrm{a}$ and $\mathrm{b}$ increased by $7 \%$ and $6 \%$ in shrubs of treated soil compared with shrubs of untreated soil in both seasons. For the effect of Moringa oleifera leaves extract, the maximum significant values of chlorophyll $\mathrm{a}$ and $\mathrm{b}$ in the leaves of jojoba shrubs were obtained from $30 \mathrm{~g} \mathrm{~L}^{-1}$ Moringa oleifera leaves extract while the minimum ones were obtained from the control treatment. Chlorophyll a and b were higher by $9 \%$ and $9 \%$ compared with that sprayed with distilled water. Regarding the combination treatments, the inoculated soil plus using the highest rate of Moringa oleifera leaves extract $\left(30 \mathrm{~g} \mathrm{~L}^{-1}\right.$ ) gave the maximum mean values of chlorophyll $\mathrm{a}$ and $\mathrm{b}$. These values increased by $16 \%$ and $17 \%$ for chlorophyll a and $\mathrm{b}$, respectively, compared with the control treatment in the two seasons.

Table 11. The mean values of chlorophyll a and $\mathrm{b}\left(\mathrm{mg} \mathrm{g}^{-1}\right)$ of jojoba shrub as affected with ground inoculation of arbuscular mycorrhizal fungi and foliar application of Moringa oleifera leaves extract as well as their combination treatments in both seasons of the study.

\begin{tabular}{|c|c|c|c|c|}
\hline \multirow{2}{*}{ Treatment } & \multicolumn{2}{|c|}{ Chlorophyll a (mg g $\left.{ }^{-1}\right)$} & \multicolumn{2}{|c|}{ Chlorophyll b (mg g $\left.{ }^{-1}\right)$} \\
\hline & 1st Season & 2nd Season & 1st Season & 2nd Season \\
\hline \multicolumn{5}{|c|}{ Arbuscular mycorrhizal fungi $\left(\mathrm{g} \mathrm{L}^{-1}\right)$} \\
\hline Control & $0.862 \pm 0.027 b$ & $0.887 \pm 0.028 b$ & $0.405 \pm 0.013 b$ & $0.417 \pm 0.013 b$ \\
\hline 20 & $0.919 \pm 0.036 \mathrm{a}$ & $0.945 \pm 0.037 \mathrm{a}$ & $0.432 \pm 0.017 \mathrm{a}$ & $0.444 \pm 0.018 \mathrm{a}$ \\
\hline \multicolumn{5}{|c|}{ Moringa oleifera leaves extract $\left(\mathrm{g} \mathrm{L}^{-1}\right)$} \\
\hline Control & $0.846 \pm 0.025 \mathrm{~d}$ & $0.871 \pm 0.026 \mathrm{~d}$ & $0.398 \pm 0.012 \mathrm{~d}$ & $0.409 \pm 0.012 \mathrm{~d}$ \\
\hline 10 & $0.882 \pm 0.030 \mathrm{c}$ & $0.907 \pm 0.031 \mathrm{c}$ & $0.414 \pm 0.014 \mathrm{c}$ & $0.426 \pm 0.015 c$ \\
\hline 20 & $0.908 \pm 0.034 b$ & $0.934 \pm 0.035 b$ & $0.427 \pm 0.016 b$ & $0.439 \pm 0.017 b$ \\
\hline 30 & $0.924 \pm 0.039 \mathrm{a}$ & $0.951 \pm 0.040 \mathrm{a}$ & $0.434 \pm 0.018 \mathrm{a}$ & $0.447 \pm 0.019 \mathrm{a}$ \\
\hline \multicolumn{5}{|c|}{ Combination treatments } \\
\hline (Control) & $0.824 \pm 0.008 \mathrm{~h}$ & $0.848 \pm 0.008 \mathrm{~h}$ & $0.387 \pm 0.004 \mathrm{~h}$ & $0.399 \pm 0.004 \mathrm{~h}$ \\
\hline $\mathrm{T} 1$ & $0.855 \pm 0.008 \mathrm{~g}$ & $0.880 \pm 0.008 \mathrm{~g}$ & $0.402 \pm 0.004 \mathrm{~g}$ & $0.414 \pm 0.004 \mathrm{~g}$ \\
\hline $\mathrm{T} 2$ & $0.878 \pm 0.009 \mathrm{e}$ & $0.903 \pm 0.009 \mathrm{e}$ & $0.412 \pm 0.004 \mathrm{e}$ & $0.424 \pm 0.004 \mathrm{e}$ \\
\hline T3 & $0.890 \pm 0.009 \mathrm{~d}$ & $0.916 \pm 0.009 \mathrm{~d}$ & $0.418 \pm 0.004 \mathrm{~d}$ & $0.430 \pm 0.004 \mathrm{~d}$ \\
\hline $\mathrm{T} 4$ & $0.868 \pm 0.008 \mathrm{f}$ & $0.894 \pm 0.008 \mathrm{f}$ & $0.408 \pm 0.004 \mathrm{f}$ & $0.420 \pm 0.004 \mathrm{f}$ \\
\hline T5 & $0.908 \pm 0.009 c$ & $0.935 \pm 0.009 c$ & $0.427 \pm 0.004 \mathrm{c}$ & $0.439 \pm 0.004 \mathrm{c}$ \\
\hline T6 & $0.938 \pm 0.009 \mathrm{~b}$ & $0.966 \pm 0.009 \mathrm{~b}$ & $0.441 \pm 0.004 \mathrm{~b}$ & $0.454 \pm 0.004 \mathrm{~b}$ \\
\hline $\mathrm{T} 7$ & $0.959 \pm 0.009 \mathrm{a}$ & $0.987 \pm 0.009 \mathrm{a}$ & $0.451 \pm 0.004 \mathrm{a}$ & $0.464 \pm 0.004 \mathrm{a}$ \\
\hline
\end{tabular}

Data are mean value \pm SE. Means in columns followed by the same letter are not statistically different at the 0.05 significance level. 


\subsection{Macro Elements (N, P and K)}

Nitrogen, phosphorus and potassium in jojoba leaves were measured and Table 12 show these results. The application of arbuscular mycorrhizal fungi affected significantly nitrogen, phosphorus and potassium content in the leaves of jojoba shrubs. Nitrogen, phosphorus and potassium percentage in the inoculated shrub was higher than that of uninoculated shrubs by $6 \%, 12 \%$ and $3 \%$ in both seasons. Moringa oleifera leaves extracts significantly increased $\mathrm{N}, \mathrm{P}, \mathrm{K}$ percentage in jojoba leaves. The application of $30 \mathrm{~g} \mathrm{~L}^{-1}$ Moringa oleifera leaves extract recorded the maximum mean percentage of nitrogen ( $9 \%)$, phosphorus $(18 \%)$ and potassium $(6 \%)$ in jojoba leaves in both seasons. Likewise for the combined effect, the treatment of arbuscular mycorrhizal fungi $\left(20 \mathrm{~g} \mathrm{~L}^{-1}\right)$ plus $30 \mathrm{~g} \mathrm{~L}^{-1}$ Moringa oleifera leaves extract recorded the maximum significant nitrogen, phosphorus and potassium in jojoba leaves in both seasons. In addition, it recorded the maximum increment percentage compared with the control treatment for nitrogen $(16 \%)$, phosphorus $(33 \%)$ and potassium $(9 \%)$ in both seasons.

Table 12. The mean values of nitrogen (\%), phosphorus (\%) and potassium percentage (\%) of jojoba shrubs affected with ground inoculation of arbuscular mycorrhizal fungi and foliar application of Moringa oleifera leaves extract, as well as their combination treatments in both seasons of the study.

\begin{tabular}{|c|c|c|c|c|c|c|}
\hline \multirow{2}{*}{ Treatment } & \multicolumn{2}{|c|}{ N (\%) } & \multicolumn{2}{|c|}{$\mathrm{P}_{2} \mathrm{O}_{5}(\%)$} & \multicolumn{2}{|c|}{$\mathrm{K}_{2} \mathrm{O}(\%)$} \\
\hline & 1st Season & 2nd Season & 1st Season & 2nd Season & 1st Season & 2nd Season \\
\hline \multicolumn{7}{|c|}{ Arbuscular mycorrhizal fungi $\left(\mathrm{g} \mathrm{L}^{-1}\right)$} \\
\hline Control & $2.742 \pm 0.122 b$ & $2.822 \pm 0.125 \mathrm{~b}$ & $0.379 \pm 0.013 \mathrm{~b}$ & $0.390 \pm 0.013 b$ & $2.665 \pm 0.062 b$ & $2.743 \pm 0.064 b$ \\
\hline 20 & $2.917 \pm 0.085 \mathrm{a}$ & $3.002 \pm 0.087 \mathrm{a}$ & $0.425 \pm 0.041 \mathrm{a}$ & $0.437 \pm 0.042 \mathrm{a}$ & $2.758 \pm 0.065 \mathrm{a}$ & $2.838 \pm 0.066 \mathrm{a}$ \\
\hline \multicolumn{7}{|c|}{ Moringa oleifera leaves extract $\left(\mathrm{g} \mathrm{L}^{-1}\right)$} \\
\hline Control & $2.683 \pm 0.121 \mathrm{~d}$ & $2.761 \pm 0.124 \mathrm{~d}$ & $0.372 \pm 0.009 \mathrm{~d}$ & $0.383 \pm 0.009 \mathrm{~d}$ & $2.632 \pm 0.046 \mathrm{~d}$ & $2.708 \pm 0.047 \mathrm{~d}$ \\
\hline 10 & $2.807 \pm 0.121 \mathrm{c}$ & $2.889 \pm 0.124 \mathrm{c}$ & $0.388 \pm 0.017 \mathrm{c}$ & $0.400 \pm 0.018 c$ & $2.688 \pm 0.072 \mathrm{c}$ & $2.767 \pm 0.073 c$ \\
\hline 20 & $2.905 \pm 0.083 b$ & $2.989 \pm 0.085 b$ & $0.408 \pm 0.027 b$ & $0.420 \pm 0.028 \mathrm{~b}$ & $2.750 \pm 0.051 \mathrm{~b}$ & $2.830 \pm 0.052 b$ \\
\hline 30 & $2.925 \pm 0.072 \mathrm{a}$ & $3.010 \pm 0.074 \mathrm{a}$ & $0.440 \pm 0.048 \mathrm{a}$ & $0.453 \pm 0.049 a$ & $2.776 \pm 0.056 \mathrm{a}$ & $2.857 \pm 0.058 \mathrm{a}$ \\
\hline \multicolumn{7}{|c|}{ Combination treatments } \\
\hline Control & $2.575 \pm 0.025 \mathrm{~h}$ & $2.650 \pm 0.025 \mathrm{~h}$ & $0.365 \pm 0.004 \mathrm{~h}$ & $0.375 \pm 0.004 \mathrm{~h}$ & $2.596 \pm 0.025 \mathrm{~h}$ & $2.671 \pm 0.025 \mathrm{~h}$ \\
\hline $\mathrm{T} 1$ & $2.699 \pm 0.026 \mathrm{~g}$ & $2.777 \pm 0.026 \mathrm{~g}$ & $0.373 \pm 0.004 \mathrm{~g}$ & $0.384 \pm 0.004 \mathrm{~g}$ & $2.627 \pm 0.026 \mathrm{~g}$ & $2.703 \pm 0.026 \mathrm{~g}$ \\
\hline $\mathrm{T} 2$ & $2.833 \pm 0.028 \mathrm{e}$ & $2.915 \pm 0.028 \mathrm{e}$ & $0.383 \pm 0.004 \mathrm{e}$ & $0.394 \pm 0.004 \mathrm{e}$ & $2.709 \pm 0.026 \mathrm{e}$ & $2.788 \pm 0.026 \mathrm{e}$ \\
\hline T3 & $2.863 \pm 0.028 \mathrm{~d}$ & $2.947 \pm 0.028 \mathrm{~d}$ & $0.397 \pm 0.004 \mathrm{~d}$ & $0.408 \pm 0.004 \mathrm{~d}$ & $2.730 \pm 0.027 \mathrm{~d}$ & $2.809 \pm 0.027 \mathrm{~d}$ \\
\hline $\mathrm{T} 4$ & $2.791 \pm 0.027 \mathrm{f}$ & $2.873 \pm 0.027 \mathrm{f}$ & $0.379 \pm 0.004 \mathrm{f}$ & $0.390 \pm 0.004 \mathrm{f}$ & $2.668 \pm 0.026 \mathrm{f}$ & $2.745 \pm 0.026 \mathrm{f}$ \\
\hline T5 & $2.915 \pm 0.028 c$ & $3.000 \pm 0.028 c$ & $0.404 \pm 0.004 \mathrm{c}$ & $0.416 \pm 0.004 c$ & $2.750 \pm 0.027 c$ & $2.830 \pm 0.027 c$ \\
\hline T6 & $2.977 \pm 0.029 \mathrm{~b}$ & $3.063 \pm 0.029 b$ & $0.433 \pm 0.004 b$ & $0.445 \pm 0.004 b$ & $2.791 \pm 0.027 b$ & $2.873 \pm 0.027 b$ \\
\hline $\mathrm{T} 7$ & $2.987 \pm 0.029 \mathrm{a}$ & $3.074 \pm 0.029 \mathrm{a}$ & $0.484 \pm 0.005 \mathrm{a}$ & $0.498 \pm 0.005 \mathrm{a}$ & $2.822 \pm 0.027 \mathrm{a}$ & $2.904 \pm 0.027 \mathrm{a}$ \\
\hline
\end{tabular}

Data are mean value \pm SE. Means in columns followed by the same letter are not statistically different at the 0.05 significance level.

\section{Discussion}

\subsection{Effect of Arbuscular Mycorrhizal Fungi}

By comparing between inoculated and uninoculated jojoba shrubs with arbuscular mycorrhizal fungi, it was noticed that inoculated shrubs were outperformed in all studied parameters of growth, flowering, fruits set, chlorophyll a and b content and N, P, K percentages. Arbuscular mycorrhizal fungus application increases photosynthesis efficiency and chlorophyll pigment content to increase biomass production [44,45]. Meanwhile, it improves flowering and fruits set. This improvement is due to the role of arbuscular mycorrhizal fungi in increasing absorption of water and nutrients, and enhances transport of immobile mineral elements through exploring larger volumes of the soil by mycorrhizal hyphae to stimulate the growth of plants [46]. These results are in harmony with studies by Davies et al. [17] on pepper, Ruiz-Lozano and Azcon [18] on lettuce, Auge et al. [19] on the common bean, Ruiz-Lozano et al. [20] on soybean, Ortas [21] on maize, wheat and cotton, Roldan et al. [23] on juniperus, Ortas and Rattan [24] on pepper, wheat and 
maize, Tobar et al. [25] on lettuce, Igiehon et al. [26] on soybean, Jabborova et al. [27] on spinach, Jabborova et al. [28] on ginger, Zewail et al. [29] on stevia and Ortas [47] on twelve plant species.

Despite the decreasing carbohydrate percentage in seeds, all the minerals, proteins and fixed oil percentages were significantly increased in treated shrubs compared with the control (uninoculated shrubs). This may be due to the role of arbuscular mycorrhizal fungus as a biostimulant in increasing the levels of macronutrients in the plant. On the other hand, the mycorrhizal symbiosis reduced the carbohydrate content of the plants compared with the control plants as it receives between 4 and $20 \%$ of photosynthetically fixed carbon from the host plant [48]. Moreover, the fungus provides the host plant with nutrients and stimulates the plant metabolism to build a high level of protein content, which may cover the plant requirements and boost the immune system, thus increasing the abiotic and biotic stress resistance of the host plant [49-51]. These results are supported by Plenchette et al. [52], who reported that the benefits of arbuscular mycorrhizal fungus are mainly attributed to improving phosphorous nutrition. Both Van der Heijden et al. [53] and Allen [54] found that arbuscular mycorrhizal fungi improve plant productivity, plant nutrition, soil structure and water uptake under semi-arid conditions. Auge [55], AlKaraki [16], Javaid [10], Khaosaad et al. [56] and Azcón et al. [57] found that arbuscular mycorrhizal fungi induced plant hormone production to increase plant photosynthesis, improve osmotic adjustment under normal, drought and salinity stresses and increase resistance to pests and soil-borne diseases against biotic and abiotic stresses.

Seed yield and oil yield of jojoba shrubs attributed to arbuscular mycorrhizal fungus in this study may be related to the role of the fungus in biostimulantion of increasing levels of macronutrients in the plant and phosphorous nutrition [52]. The increase in yield due to phosphorous nutrition may be attributed to the activation of metabolic processes, where its role in building phospholipids and nucleic acid is known [58,59]. Phosphorous is a key constituent of ATP and plays a significant role in energy transformation in plant and also plays a role in seed formation. Application of $\mathrm{P}, \mathrm{Ca}$ and $\mathrm{B}$ fertilizers increased nutrient availability to the crops during the growing season, which leads to greater utilization of assimilates into the fruits and ultimately increased the number of filled fruits and set percentage [60]. Therefore, oil content in seeds increased gradually with increasing levels of phosphorus [59,61,62]. Priya et al. [63] and Atteya et al. [64] reported that the increase in oil content and oil yield is attributed to the application of a higher level of N, P and K to the crop. Giri et al. [65] noticed that using arbuscular mycorrhizal fungi increased the oil content of Dutch fennel plants.

\subsection{Effect of Moringa Oleifera Extract}

The increase in improvement of growth, flowering, fruits set, chlorophyll $a$ and $b$ content and N, P, K percentages was in parallel with increasing the concentration of Moringa oleifera leaves extract concentration. This may be due to the collective performance of macro and micronutrients, amino acids, vitamins, ascorbates, phenolic compounds antioxidants and growth-regulating hormones such as auxins, cytokinins, gibberellins, jasmonic and salicylic acids present in Moringa leaves extract [30-34]. This improvement in vegetative growth and flowering was reflected in the seed yield and oil yield of jojoba shrubs in this study. Fruits are the main sink of plant production. Gibberellins increase sink demand by increasing fruit cell elongation and enhancement of phloem unloading or/and metabolism of carbon assimilates in fruit, as gibberellins induce activities of sugar metabolizing enzymes. Therefore, it finally increases seed and oil yields $[6,66,67]$. Our results are in agreement with many researchers, as Moringa leaves extract has been reported to increase the growth, yield and chemical composition of many crops such as maize, rice, sorghum, wheat [7,68], tomato [69], basil [35], common bean [33], roselle [70], geranium [36], freesia [37] and fennel [34]. 


\subsection{Effect of Combination Treatments}

The increase in herb content of nitrogen, phosphorus and potassium may be due to Moringa leaves extract, which is a rich source of amino acids, potassium, calcium, iron, vitamin E, ascorbates, phenolic compounds and growth-regulating hormones such as zeatin [30,32]. Additionally, arbuscular mycorrhizal fungi improved the productivity of plants, which was attributed to enhanced uptake of immobile nutrients such as phosphorus, zinc, nitrogen and copper. This increase in the presence of zeatin of Moringa leaves extract increased chlorophyll a and $\mathrm{b}$ content and the photosynthesis process. Therefore, in this study, vegetation and flowering as well as fruits set were improved, and then the yield of seeds and oil was improved too as a result of combination treatments of biological and stimulating fertilizers. These results agree with El-Serafya and El-Sheshtawy [34] on fennel, Mervat et al. [71] on grapevines, Djouhou et al. [46] on cowpea and sorghum and Djouhou et al. [72] on Moringa. Atteya et al. [64] reported that fertilization treatments enhanced seed and oil yields of the Moringa oleifera tree and affected fatty acid percentages. Atteya et al. [6] reported that jojoba seeds contain approximately $53.68 \%$ fixed oil percentage, and the main fatty acid is Gadoleic acid (C20:1), which represents $53.54 \%$; the second, Oleic acid (C18:1), represents $15.59 \%$. Genaidy et al. [73] reported that the Strain EAI of jojoba shrubs is distinguished by a high yield of seeds and oil.

\section{Conclusions}

We can conclude from this study that the application of both mycorrhizal fungi and Moringa oleifera leaves extract is recommended to increase seed and oil yields of the jojoba shrub. Moreover, the application of combination treatment of $20 \mathrm{~g} \mathrm{~L}^{-1}$ mycorrhizal fungi plus $30 \mathrm{~g} \mathrm{~L}^{-1}$ Moringa oleifera leaves extract recorded the maximum mean values of main branch length, length of secondary branches, number of branched nodes, number of secondary branches, flowering percentage, final fruit set percentage, seed yield per shrub and per hectare, percentage of minerals, proteins as well as oil yield per shrub and per hectare, chlorophyll a and $b, N, P, K$ percentage with a minimum mean value of a number of days until full bloom in both seasons. The maximum percentage of gadoleic fatty acid was found with the combination treatment of $0 \mathrm{~g} \mathrm{~L}^{-1}$ mycorrhizal fungi plus $10 \mathrm{~g} \mathrm{~L}^{-1}$ Moringa oleifera leaves extract.

Author Contributions: Conceptualization, A.K.G.A. and E.A.E.G.; methodology, A.K.G.A. and E.A.E.G.; software, A.K.G.A., R.S., A.A.M.A.-M., K.A.I., E.A.E.G.; validation, A.K.G.A., R.S., A.A.M.A.-M., K.A.I., E.A.E.G.; formal analysis, A.K.G.A. and E.A.E.G.; investigation, A.K.G.A. and E.A.E.G.; resources, A.K.G.A., R.S., A.A.M.A.-M., K.A.I., E.A.E.G.; data curation, A.K.G.A. and E.A.E.G.; writing—original draft preparation, A.K.G.A. and E.A.E.G.; writing-review and editing, A.K.G.A. and E.A.E.G.; visualization, A.K.G.A. and E.A.E.G.; supervision, A.K.G.A. and E.A.E.G. funding acquisition, R.S., A.A.M.A.-M., K.A.I.; All authors have read and agreed to the published version of the manuscript.

Funding: The study did not receive any external financing.

Institutional Review Board Statement: Not applicable.

Informed Consent Statement: Not applicable.

Data Availability Statement: The data presented in this study are available within the article.

Acknowledgments: The authors would like to thank the Faculty of Agriculture, Damanhour University, Egypt. Taif University Researchers Supporting Project Number (TURSP-2020/140), Taif University, Taif, Saudi Arabia. Additionally, the authors thank Prince Sattam Bin Abdulaziz University, Al-Kharj for their scientific contributions. The authors would like to thank Murthy Chavali Office of the Dean (Research) and Division of Chemistry, Department of Science, Faculty of Science and Technology, Alliance University, Chikkahagade Cross, Chandapura-Anekal Main Road, Bengaluru 562106 Karnataka, India for revision of this article.

Conflicts of Interest: The authors declare no conflict of interest. 


\section{References}

1. Nelson, J.M.; Watson, J.E. Nitrogen fertilization effects on jojoba seed production. Ind. Crops Prod. 2001, 13, 145-154. [CrossRef]

2. Phillips, S.J.; Patricia, W.C. (Eds.) A Natural History of the Sonoran Desert; University of California Press: Berkeley, CA, USA, 2001; pp. 256-257, ISBN 0-520-21980-5.

3. Wisniak, J. The Chemistry and Technology of Jojoba Oil; American Oil Chemists' Society: Champaign, IL, USA, 1987.

4. US National Research Council. Jojoba: New Crop for Arid Lands, New Material for Industry; National Academy Press: Washington, DC, USA, 1985.

5. Guardiola, J.L. Fruit set and growth. In Second International Seminar on Citrus; Donadio, L.C., Ed.; University of Bebedouro: Bebedouro, Brazil, 1992; pp. 1-30.

6. Atteya, A.K.G.; Genaidy, E.A.E.; Zahran, H.A. Chemical constituents and yield of Simmondsia chinensis plants as affected by foliar application of gibberellic acid and zinc sulphate. Biosci. Res. 2018, 15, 1528-1541.

7. Phiri, C. Influence of Moringa oleifera leaf extracts on germination and early seedling development of major cereals. ABJNA 2010, 1, 774-777. [CrossRef]

8. Raja, N. Biopesticides and biofertilizers: Ecofriendly sources for sustainable agriculture. J. Biofertil. Biopestici. 2013,4 , e112. [CrossRef]

9. Hooker, J.E.; Blackke, E. Arbuscular mycorrhizal fungi as components of sustainable soil-plant systems. Crit. Rev. Biotechnol. 1995, 15, 201-212. [CrossRef]

10. Javaid, A. Allelopathic interactions in mycorrhizal associations. Allelopath. J. 2007, 20, $29-42$.

11. Javaid, A.; Jabeen, K.; Javaid, A. Effect of NPK fertilizers and two types of green manures on growth and mycorrhizal colonization of wheat. Pak. J. Phytopathol. 2007, 19, 132-138.

12. Javaid, A.; Riaz, T. Mycorrhizal colonization in different varieties of Gladiolus and its relation with plant vegetative and reproductive growth. Int. J. Agric. Biol. 2008, 10, 278-282.

13. Smith, S.E.; Read, D.J. Mycorrhizal Symbiosis, 3rd ed.; Academic Press: London, UK, 2008.

14. Entry, J.A.; Rygiewicz, P.T.; Watrud, L.S.; Donnelly, P.K. Influence of adverse soil conditions on the formation and function of Arbuscular mycorrhizas. Adv. Environ. Res. 2002, 7, 123-138. [CrossRef]

15. Javaid, A. Arbuscular mycorrhizal mediated nutrition in plants. J. Plant Nutr. 2009, 32, 1595-1618. [CrossRef]

16. Al-Karaki, G.N. Nursery inoculation of tomato with Arbuscular Mycorrhizal Fungi and subsequent performance under irrigation with saline water. Sci. Hort. 2006, 109, 1-7. [CrossRef]

17. Davies, F., Jr.; Potter, J.; Linderman, R. Mycorrhiza and repeated drought exposure affect drought resistance and extraradical hyphae development of pepper plants independent of plant size and nutrient content. J. Plant Physiol. 1992, 139, 289-294. [CrossRef]

18. Ruiz-Lozano, J.; Azcon, R. Hyphal contribution to water uptake in mycorrhizal plants as affected by the fungal species and water status. Physiol. Plantar. 1995, 95, 472-478. [CrossRef]

19. Auge, R.M.; Moore, J.L.; Cho, K.; Stutz, J.C.; Sylvia, D.M.; Al-Agely, A.K.; Saxton, A.M. Relating foliar dehydration tolerance of mycorrhizal Phaseolus vulgaris to soil and root colonization by hyphae. J. Plant Physiol. 2003, 160, 1147-1156. [CrossRef] [PubMed]

20. Ruiz-Lozano, J.M.; Collados, C.; Barea, J.M.; Azcon, R. Arbuscular mycorrhizal symbiosis can alleviate drought-induced nodule senescence in soybean plants. New Phytol. 2001, 151, 493-502. [CrossRef]

21. Ortas, I. Effect of selected mycorrhizal inoculation on phosphorus sustainability in sterile and non-sterile soils in the Harran Plain in South Anatolia. J. Plant Nutr. 2003, 26, 1-17. [CrossRef]

22. Karandashov, V.; Bucher, M. Symbiotic phosphate transport in Arbuscular Mycorrhizas. Trends Shrub Sci. 2005, 10, 22-29. [CrossRef]

23. Roldan, A.; Díaz-Vivancos, P.; Hernandez, J.A.; Carrasco, L.; Caravaca, F. Superoxide dismutase and total peroxidase activities in relation to drought recovery performance of mycorrhizal shrub seedlings grown in an amended semiarid soil. J. Plant Physiol. 2008, 165, 715-722. [CrossRef]

24. Ortas, I.; Lal, R. Long-Term Fertilization Effect on Agronomic Yield and Soil Organic Carbon under Semi-Arid Mediterranean Region. Am. J. Exp. Agric. 2014, 4, 1086-1102. [CrossRef]

25. Tobar, R.M.; Azcon, R.; Barea, J.M. Improved nitrogen uptake and transport from 15N-labeled nitrate by external hyphae of arbuscular mycorrhiza under water-stressed conditions. New Phytol. 1994, 126, 119-122. [CrossRef]

26. Igiehon, N.O.; Babalola, O.O.; Cheseto, X.; Torto, B. Effects of rhizobia and Arbuscular Mycorrhizal Fungi on yield, size, distribution and fatty acid of soybean seeds grown under drought stress. Microbiol. Res. 2021, 242, 126640. [CrossRef] [PubMed]

27. Jabborova, D.; Annapurna, K.; Paul, S.; Kumar, S.; Saad, H.A.; Desouky, S.; Ibrahim, M.F.M.; Elkelish, A. Beneficial features of biochar and Arbuscular Mycorrhiza for improving spinach shrub growth, root morphological traits, physiological properties, and soil enzymatic activities. JoF 2021, 7, 571. [CrossRef]

28. Jabborova, D.; Wirth, S.; Halwani, M.; Ibrahim, M.F.M.; Azab, I.H.E.; El-Mogy, M.M.; Elkelish, A. Growth response of Ginger (Zingiber Officinale), its physiological properties and soil enzyme activities after biochar application under greenhouse conditions. Horticulturae 2021, 7, 250. [CrossRef]

29. Zewail, R.M.Y.; Ali, M.; El-Gamal, I.S.H.; Al-Maracy, S.H.A.; Islam, K.R.; Elsadek, M.; Azab, E.; Gobouri, A.A.; ElNahhas, N.; Mohamed, M.H.M.; et al. Interactive Effects of Arbuscular Mycorrhizal Inoculation with Nano Boron, Zinc, and Molybdenum Fertilization on Stevioside Contents of Stevia (Stevia rebaudiana, L.) Shrubs. Horticulturae 2021, 7, 260. [CrossRef] 
30. Makkar, H.P.S.; Becker, K. Nutritional value and antinutritional components of whole and ethanol extracted Moringa oleifera leaves. Anim. Feed Sci. Technol. 1996, 63, 211-288. [CrossRef]

31. Nambiar, V.S.; Rachana, M.; Daniel, M. Polyphenol content of three Indian green leafy vegetables. J. Food Sci. Technol. 2005, 42, 312-315.

32. Nagar, P.K.; Iyer, R.I.; Sircar, P.K. Cytokinins in developing fruits of Moringa pterigosperma Gaertn. Physiol. Plant. 2006, 55, 45-50. [CrossRef]

33. Rady, M.M.; Mohamed, G.F. Modulation of salt stress effects on the growth, physio-chemical attributes and yields of Phaseolus vulgaris L. plants by the combined application of salicylic acid and Moringa oleifera leaf extract. Sci. Hort. 2015, 193, 105-113. [CrossRef]

34. El-Serafya, R.S.; El-Sheshtawy, A.A. Effect of nitrogen fixing bacteria and moringa leaf extract on fruit yield, estragole content and total phenols of organic fennel. Sci. Hortic. 2020, 265, 109209. [CrossRef]

35. Prabhu, M.; Kumar, A.R.; Rajamani, K. Influence of different organic substances on growth and herb yield of sacred basil (Ocimum sanctum L.). Indian J. Agric. Res. 2010, 44, 48-52.

36. Ali, E.F.; Hassan, F.A.S.; Elgimabi, M. Improving the growth, yield and volatile oil content of Pelargonium graveolens L. Herit by foliar application with Moringa leaf extract through motivating physiological and biochemical parameters. S. Afr. J. Bot. 2018, 119, 383-389. [CrossRef]

37. Ahmad, I.; Tanveer, M.U.; Liaqat, M.; Doleb, J.M. Comparison of corm soaks with preharvest foliar application of moringa leaf extract for improving growth and yield of cut Freesia hybrid. Sci. Hortic. 2019, 254, 21-25. [CrossRef]

38. Peter, K.V. Handbook of Herbs and Spice 2; Elsevier, Imprint Woodhead Publishing: Cambridge, UK, 2012 ; pp. $275-302$.

39. Dayananda, B.; Raghavan, A.K.; Khanum, F.; Singh, B.A. In vitro antioxidant and free radical scavenging activity of Glycyrrhiza glabra root extracts. J. Herbal Med. Toxicol. 2010, 4, 97-102.

40. AOAC. Official Methods of Analysis, 15th ed.; Association Official Analytical Chemist: Arlington, TX, USA, 1995.

41. Wintermans, J.F.G.M.; Mats, D.E. Spectrophtometeric characteristic of chlorophyll and their pheophytins in ethanol. Biochem. Biophys. Acta. 1965, 109, 448-453.

42. Chapman, H.D.; Pratt, P.F. Methods of Analysis for Soil Plant and Water; California University USA: Oakland, CA, USA, 1961.

43. SAS Institute Inc. SAS/STAT User's Guide, Release 6.03 Edition; SAS Institute: Cary, NC, USA, 1988.

44. Wright, D.P.; Scholes, J.D.; Read, D.J. Effects of VA mycorrhizal colonization on photosynthesis and biomass production of Trifolium repens L. Plant Cell Environ. 1998, 21, 209-216. [CrossRef]

45. Mandal, S.; Evelin, H.; Giri, B.; Singh, V.P.; Kapoor, R. Arbuscular Mycorrhiza enhances the production of stevioside and rebaudioside-A in Stevia rebaudiana via nutritional and non-nutritional mechanisms. Appl. Soil Ecol. 2013, 72, 187-194. [CrossRef]

46. Djouhou, F.M.C.; Fokou, E.; Nwaga, D. Potential of Moringa oleifera leaf powder and beneficial microorganisms (Mycorrhizal fungi and rhizobia) in modulating plant symbiosis and yield. Sch. J. Agric. Vet. Sci. 2018, 5, 236-243.

47. Ortas, I. The effect of Mycorrhizal Fungal inoculation on plant yield, nutrient uptake and inoculation effectiveness under long-term field conditions. Field Crops Res. 2012, 125, 35-48. [CrossRef]

48. Müller, V.; Spanheimer, R.; Santos, H. Stress response by solute accumulation in archaea. Curr. Opin. Microbiol. 2005, 8, 729-736. [CrossRef] [PubMed]

49. Kyriazakis, I.; Houdijk, J.G. Nutritional control of parasites. Small Rumin. Res. 2006, 62, 79-82. [CrossRef]

50. Brisibe, E.A.; Umoren, U.E.; Brisibe, F.; Magalhaes, P.M.; Ferreira, J.F.S.; Luthria, D.; Wu, X.; Prior, R.L. Nutritional Characterization and Antioxidant Capacity of Different Tissues of Artemisia annua L. Food Chem. 2009, 115, 1240-1246. [CrossRef]

51. Adewole, M.B.; Ilesanmi, A.O. Effects of soil amendments on the nutritional quality of okra (Abelmoschus esculentus [L.] Moench). J. Soil Sci. Plant Nutr. 2011, 11, 45-55.

52. Plenchette, C.; Clermont-Dauphenic, C.; Meynard, J.M.; Fortin, J.A. Managing arbuscular mycorrhizal fungi in cropping systems. Can. J. Plant Sci. 2005, 85, 31-40. [CrossRef]

53. Van der Heijden, M.G.A.; Streitwolf-Engel, R.; Riedl, R. The mycorrhizal contribution to plant productivity, plant nutrition and soil structure in experimental grassland. New Phytologist. 2006, 172, 739-752. [CrossRef]

54. Allen, M.F. Linking water and nutrients through the vadose zone: A fungal interface between the soil and plant systems. Arid Land 2011, 3, 155-163. [CrossRef]

55. Auge, R.M. Water relations, drought and Vesicular-Arbuscular Mycorrhizal symbiosis. Mycorrhiza 2001, 11, 3-42. [CrossRef]

56. Khaosaad, T.; García-Garrido, J.M.; Steinkellner, S.; Vierheilig, H. Takeall disease is systemically reduced in roots of mycorrhizal barley plants. Soil Biol. Biochem. 2007, 39, 727-734. [CrossRef]

57. Azcón, R.; Perálvarez, M.C.; Biró, B.; Roldán, A.; Ruíz-Lozano, J.M. Antioxidant activities and metal acquisition in mycorrhizal plants growing in a heavy-metal multicontaminated soil amended with treated lingo cellulosic agrowaste. Appl. Soil Ecol. 2009, 41, 168-177. [CrossRef]

58. Singh, R.V.; Singh, M.P. Response of safflower to moisture regimes, plant population and phosphorus. Indian J. Agron. 1989, 34, 88-91.

59. Kumari, M.S.; Saritha, J.D. Effect of phosphorus fertilizers on oil seed crops. Agric. Update 2017, 12, 749-754. [CrossRef]

60. Rezaul, K.; Sabina Yeasmin, A.K.M.; Islam, M.; Sarkar, R. Effect of phosphorus, calcium and boron on the growth and yield of groundnut (Arachis hypogea L.). Internat. J. Bio-Sci. Bio-Technol. 2013, 5, 51-60. 
61. Jones, P.; Sreenivasa, M.N. Response of sunflower to the inoculation of VA mycorrhiza and or phosphate solubilising bacteria in black clayey soil. J. Oilseeds Res. 1993, 10, 86-92.

62. Mishra, A.; Das, P.; Paikaray, R.K. Performance of sunflower in relation to nitrogen and phosphorus in acid soils of Orissa. J. Oilseeds Res. 1994, 11, 288-290.

63. Priya, S.R.; Mohammed, M.; Maheswari, J.; Sangeetha, S.P. Influence of NPK fertilizers on productivity and oil yield of groundnut(Arachis hypogaea) and sunflower (Helianthus annuus)in intercropping system under irrigated condition. Internat. J. Agric. Res. 2009, 4, 97-106. [CrossRef]

64. Atteya, A.K.G.; Albalwa, A.N.; El-Serafy, R.S.; Albalwa, K.; Bayomy, H.M.; Genaidy, E.A.E. Respose of Moringa oleifera seeds and fixed oil production to vermicompost and NPK under calcareous soil conditions. Plants 2021, 10, 1998. [CrossRef]

65. Giri, B.; Kapoor, R.; Mukerji, K.G. Influence of arbuscular mycorrhizal fungi and salinity on growth, biomass, and mineral nutrition of Acacia auriculiformis. Biol. Fertil. Soils 2003, 38, 170-175. [CrossRef]

66. Marschner, H. Mineral Nutrition of Higher Plants; Academic Press: London, UK, 1986; p. 674.

67. Eman, A.A.; Abd El-moneim, M.M.M.; Abd El Migeed, O.; Ismail, M.M. GA3 and Zinc Sprays for improving yield and fruit quality of washington navel orange trees grown under sandy soil conditions. Res. J. Agric. Biol. Sci. 2007, 3, 498-503.

68. Abbas, R.N.; Tanveer, A.; Khaliq, A.; Iqbal, A.; Ghaffari, A.R.; Matloob, A.; Maqsood, Q. Maize (Zea mays L.) germination, growth and yield response to foliar application of Moringa oleifera Lam. Leaf extracts. Crop Environ. 2013, 4, 39-45.

69. Culver, M.; Fanuel, T.; Chiteka, A.Z. Effect of Moringa extract on growth and yield of tomato. Greener J. Agric. Sci. 2012, 2, 207-211.

70. Hassan, H.M.S.; Abd El-Samee, M.A.S. Growth, yield and nutritional value of Hibiscus sabdariffa L. as influenced by licorice and Moringa aqueous extracts under North Sinai conditions. Zagazig J. Agric. Res. 2015, 42, 1069-1079.

71. Mervat, A.A.; Shawky, S.M.; Shaker, G.S. Comparative efficacy of some bioagents, plant oil and plant aqueous extracts in controlling Meloidogyne incognita on growth and yield of grapevines. Ann. Agric. Sci. 2012, 57, 7-18. [CrossRef]

72. Djouhou, F.M.C.; Nwaga, D.; Fokou, E. Comparative Effect of Arbuscular Mycorrhizal Fungi and Biostimulants on the Antioxidant and Nutritional Potential of Moringa oleifera. Nutri. Food Sci. Int. J. 2019, 9, 555758. [CrossRef]

73. Genaidy, E.A.E.; Atteya, A.K.G.; Adss, I.A.A. Increase the economic value of the jojoba (Simmondsia chinensis) yield using evaluation of distinctive clones grown under the Egyptian environmental conditions. J. Agric. Sci. Technol. 2016, 12, 145-165. 